\title{
Modulating the photoluminescence of conducting polymer by the surface plasmon of Au colloids
}

\author{
I-Shuo Liu ${ }^{\mathrm{a}}$, Yang-Fang Chen ${ }^{\mathrm{b}, \mathrm{c}, 1}$, Wei-Fang Su ${ }^{\mathrm{a}, \mathrm{c}, \mathrm{d}, *}$ \\ a Department of Materials Science and Engineering, National Taiwan University, Taipei, Taiwan \\ ${ }^{\mathrm{b}}$ Department of Physics, National Taiwan University, Taipei, Taiwan \\ c Center for Condensed Matter Science, National Taiwan University, Taipei, Taiwan \\ d Graduate Institute of Polymer Science and Engineering, National Taiwan University, Taipei, Taiwan
}

\section{A R T I C L E I N F O}

\section{Article history:}

Received 28 December 2007

Received in revised form 1 April 2008

Accepted 6 June 2008

Available online 20 June 2008

\section{Keywords:}

Surface plasmon

Conducting polymer

Resonance

Colloid

Photoluminescence

\begin{abstract}
A B S T R A C T
The modulation of photoluminescence of conducting polymers using Au colloids was studied for poly(3hexyl thiophene) (P3HT) and poly(2-methoxy-5-(2-ethyl)(hexyloxy) 1,4-phenylenevinylene) (MEHPPV). The extent of modulation can be explained by the extinction of Au colloids and quantum yield of polymer solution. Two types of Au colloids were investigated: nanocluster $(\sim 100 \mathrm{~nm})$ and nanoparticles $(\sim 5 \mathrm{~nm})$. The addition of the Au nanocluster into either one of the polymer solutions ( $0.08 \mathrm{wt} . \%)$ increases the photoluminescence (PL) of each polymer due to the scattering effect from the Au surface plasmon resonance. In contrast, the addition of Au nanoparticle which surface plasmon resonance comes from the absorption component causes a PL quenching. We have observed the PL intensity remains unchanged for P3HT but decreases for MEHPPV. The low quantum yield characteristic of P3HT has stronger polymer interactions than that of MEHPPV, this reduces energy transfer to Au nanoparticles, then dissipates further into heat with no decrease in PL. The polymer interactions are minimized using a dilute polymer solution $\left(1.48 \times 10^{-5} \mathrm{wt} . \%\right.$ for MEHPPV and P3HT respectively). In this case, the nonradiative decay rate of Au colloids becomes dominant, the decrease of PL is observed for both polymers. The photoluminescence of conducting polymer can be modulated by controlling the interactions among polymers and Au colloids. (c) 2008 Elsevier B.V. All rights reserved.
\end{abstract}

\section{Introduction}

Conducting polymers have attracted a great deal of attention because of their potential for fabricating large area and flexible electronics such as in polymer light-emitting diodes (PLED). Improving the fluorescence intensity of these conducting polymers has become a very important issue in the field of PLED. Recently, localized surface plasmons excited on metal particles have come under intense scrutiny, because they greatly enhance local electromagnetic field near the surfaces of particles. Surface-enhanced Raman scattering (SERS), in particular, exploits such large local fields, e.g., in the study of molecules [1-5] or polymers [6] adsorbed on metal nanoparticles or on rough surfaces of $\mathrm{Au}, \mathrm{Ag}$, and $\mathrm{Cu}$. However, in contrast to the inelastic Raman process, the details of

\footnotetext{
* Corresponding author at: Department of Materials Science and Engineering, National Taiwan University, No. 1, Sec. 4, Roosevelt Rd., Taipei, 106, Taiwan. Tel.: +8862 33664078; fax: +886233664078.

E-mail addresses: yfchen@phys.ntu.edu.tw(Y.-F. Chen), suwf@ntu.edu.tw (W.-F. Su).

1 Tel.: +886233665125.
}

the role that surface plasmons play in photoexcited fluorescence involving surface plasmons and emitter (e.g. dyes [7-12], semiconductors [13-15], and conducting polymers [16]) is still under debate. A competition exists between the enhancement of a local field and the energy transfer from the excited emitter to the metal particles or film. This phenomenon is complex and requires further investigation by experimentation and detailed analysis. The interplay is expected to depend strongly on the size and shape of the metal and emitter. However, experimental reports from many different particle arrangements vary widely in terms of reported fluorescence enhancement or quenching and are not always accompanied by fully transparent physical arguments. Although organic elements are very important materials in the fabrication of light emitting diode (LED), there are few discussions in the literature regarding the photoluminescence (PL) phenomena between the conducting polymers and their surface plasmon resonances [16]. Instead, many reports focus on the optical properties between dyes and metals [7-12].

Here we report the PL properties of conducting polymers that include Au colloids. Previous reports suggest the enhancement of fluorescence intensity strongly depends on the quantum efficiency (QE) of the dye molecule [11,12]. We have found the similar 
phenomena observed in conducting polymers. We also find that the surface plasmon coming from the dominant scattering component or absorption component has a very different influence on the PL intensity of polymer. When the plasmon resonance arises from the scattering component, the PL of conducting polymer is enhanced. In contrast, the absorption component does not enhance PL. Our experimental results are consistent with the theory proposed for dye molecule with nanostructured metal $[9,11,12]$. We have found that the control of plasmon resonance frequency of metal colloids to match with the emitting range of polymer and the quantum efficiency of polymer are especially important to realize an efficient enhancement in polymer fluorescence.

\section{Experimental}

All inorganic chemicals were purchased from Acros Chemicals (Belgium) and used without further purification. We modified O'Brien's method [17] to synthesize Au nanoparticles. A gold nanoparticle precursor solution was prepared by dissolving $0.03 \mathrm{~g}$ $\mathrm{HAuCl}_{4} \cdot 3 \mathrm{H}_{2} \mathrm{O}$ in $1-2 \mathrm{ml}$ of tri-n-butylphosphine. A reaction flask containing $6 \mathrm{~g}$ trioctylphosphine oxide (TOPO), $3 \mathrm{~g}$ hexadecylamine, and $0.1 \mathrm{~g} \mathrm{NaBH}_{4}$ was heated to $190^{\circ} \mathrm{C}$ under $\mathrm{Ar}$ environment. The precursor solution was injected quickly into a reaction flask and the temperature was also kept at $190^{\circ} \mathrm{C}$ for $30 \mathrm{~min}$. The nanoparticles were precipitated out by adding methanol $(20 \mathrm{~g})$ then redissolved in toluene. The morphology of Au nanoparticles can be controlled by the injection volume of tri-n-butylphosphine. For example, $2 \mathrm{ml}$ of tri- $n$-butylphosphine resulted in clusters of Au nanoparticles, and $1 \mathrm{ml}$ of tri- $n$-butylphosphine resulted in isolated Au nanoparticles. The P3HT and MEHPPV were ordered from Aldrich Chemical Company (U.S.A.). The P3HT was dissolved in toluene to make a 0.08 and $1.48 \times 10^{-5}$ wt.\% respectively. The MEHPPV was also dissolved in toluene to make a 0.08 and $1.48 \times 10^{-5}$ wt.\% respectively. The Au nanoparticle was added into the polymer solutions (P3HT or MEHPPV) to make the Au concentrations $3.06 \times 10^{-4}, 1.22 \times 10^{-3}, 3.67 \times 10^{-3}, 6.11 \times 10^{-3}$, and $8.56 \times 10^{-3}$ wt.\% respectively. The extinction spectra of the solution were studied by using HEXIOS- $\gamma$, Thermo Spectronic Company (U.S.A.). The PL spectra of the solutions were studied by using LS55, PerkinElmer Company (U.S.A.). The exciting wavelength of PL was $400 \mathrm{~nm}$. The morphology of Au nanoparticle was studied by transmission electron microscopy (TEM), JEM-1230, JOEL Company (Japan). Time-resolved PL spectroscopy was performed with a time-correlated single photon counting (TCSPC) spectrometer (Picoquant, Inc.). A pulse laser $(375 \mathrm{~nm})$ with an average power of $1 \mathrm{~mW}$ operating at $40 \mathrm{MHz}$ with duration of $70 \mathrm{ps}$ was used for excitation.

Quantum yields of the polymers (P3HT and MEHPPV) with $\mathrm{Au}$ clusters or $\mathrm{Au}$ nanoparticles were determined relative to 4-(dicyanomethylene)-2-methyl-6(p-dimethylamino-styryl) $4 \mathrm{H}$ pyran (DCM, laser grade, Exciton) in methanol according to the following equation[18]:

$Q=Q_{\mathrm{DCM}}\left(\frac{I}{I_{\mathrm{DCM}}}\right)\left(\frac{A_{\mathrm{DCM}}}{A}\right)\left(\frac{n^{2}}{n_{\mathrm{DCM}}^{2}}\right)$

where the quantum yield $Q_{D C M}$ for DCM in methanol is taken as 0.44 [19], I is the integrated intensity of sample over the wavelength region of $500-700 \mathrm{~nm}, I_{\mathrm{DCM}}$ is the integrated intensity of DCM in methanol over the wavelength region of $500-700 \mathrm{~nm}, A_{\mathrm{DCM}}$ is the DCM absorbance at $400 \mathrm{~nm}$ (exciting wavelength), $A$ is the sample absorbance at $400 \mathrm{~nm}, n$ is the refractive index of toluene used in sample, and $n_{\mathrm{DCM}}$ is the refractive index of methanol used in DCM.

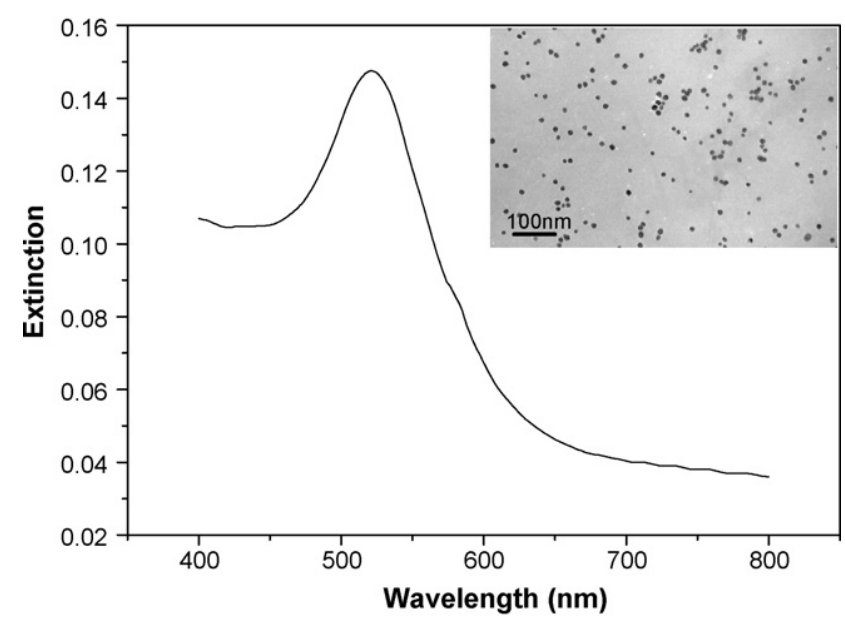

Fig. 1. Extinction spectrum of isolated Au nanoparticles, the inset shows the TEM of isolated Au nanoparticles.

\section{Results and discussion}

Two types of Au colloids were used in this study: nanoparticles and nanoclusters. The Au nanoparticles are isolated particles having sizes that range from 5 to $20 \mathrm{~nm}$ and exhibit surface plasmon resonance at $520 \mathrm{~nm}$ (Fig. 1). The Au clusters are the aggregates of $\mathrm{Au}$ nanoparticles with sizes larger than $50 \mathrm{~nm}$ (Fig. 2). When the interparticle distance is comparable to or less than the particle size, the interactions between particles play a significant role in the optical response [20-22]. The Au nanoparticles attract each other by dipole-dipole interactions when they near each other. Often these interactions are interpreted using electrostatic concepts, where the interaction of parallel collinear dipoles typically leads to redshift of the plasmon wavelengths, while parallel but noncollinear dipoles produce blue-shift plasmon wavelengths [20,21]. From various theoretical and experimental results [20-22], the full width at half maximum (FWHM) of the extinction peaks increase when the metal nanoparticles aggregate. In our experiments, the surface plasmon resonance of Au clusters is red shifted from Au nanoparticles to $\sim 600 \mathrm{~nm}$ (Fig. 2). Blue-shifted plasmon wavelengths of the noncollinear dipoles were not observed. The broad $600 \mathrm{~nm}$ peak may result from the noncollinear dipole peak overlapping with the collinear dipole peak.

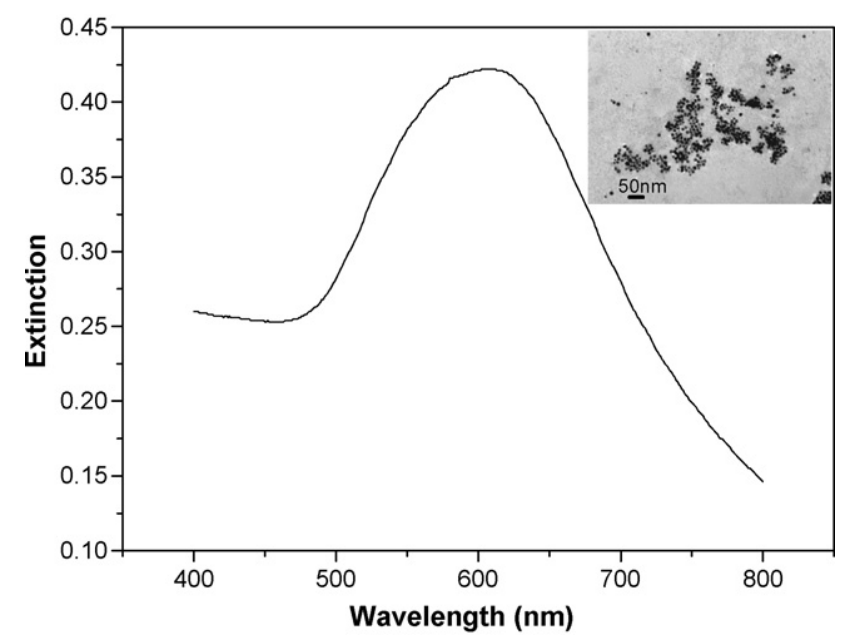

Fig. 2. Extinction spectrum of Au clusters, the inset shows the TEM image of Au clusters. 
We investigated the effects of the surface plasmon of Au clusters or Au nanoparticles on the PL intensity of conducting polymers. For the P3HT solution containing Au colloids, the concentration of the P3HT was kept at 0.08 wt.\% in toluene and the concentrations of Au clusters or Au nanoparticles were varied at $3.06 \times 10^{-4}$, $1.22 \times 10^{-3}, 3.67 \times 10^{-3}, 6.11 \times 10^{-3}$, and $8.56 \times 10^{-3} \mathrm{wt} . \%$, respectively. The PL intensity of P3HT solution containing Au clusters increased three fold over that of the pure P3HT solution (Fig. 3(a)). However, the PL intensity of P3HT did not increase when we added the isolated (unclustered) Au nanoparticles into the solution (Fig. 4(a)). Similar experiments were also done for MEHPPV. To our surprise, the addition of clusters or Au nanoparticles into the MEHPPV did not provide the same results as the P3HT. PL quenching was observed when the concentration of Au nanoparticles was increased (Fig. 4(b)). The PL intensity of MEHPPV increases initially but decreases to original intensity at certain Au cluster concentrations (Fig. 3(b)). We will explain the phenomenon why the PL enhancement of the P3HT is much stronger than that of MEHPPV in the presence of Au clusters and why the PL quenching is observed in the polymer solution containing Au nanoparticles for MEHPPV but not for P3HT in the following sections.

From the models proposed for the behaviors of fluorescence molecules with metal nanostructure systems [9,11-12,23-26],
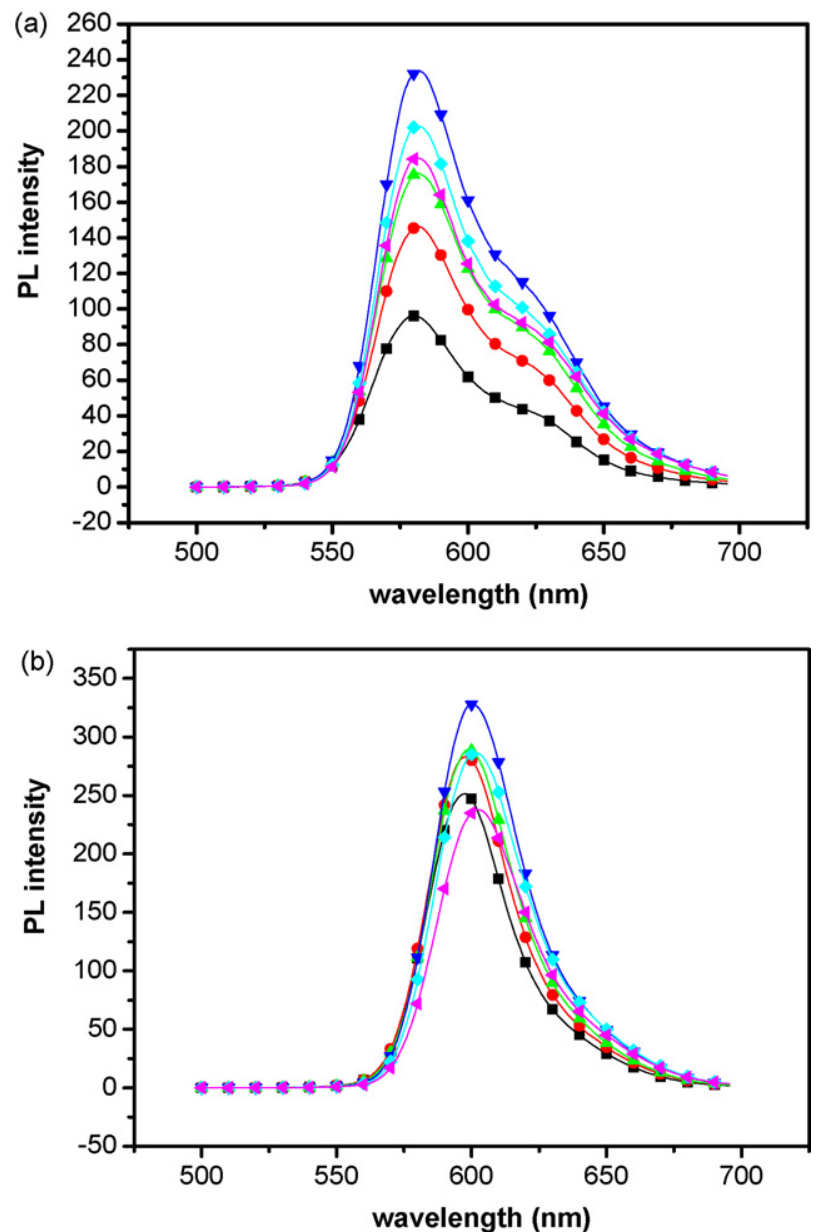

Fig. 3. PL spectra of high concentration ( 0.08 wt.\%) of (a) P3HT solution and (b) MEHPPV solution with or without Au clusters. The black curves represent pure P3HT and MEHPPV respectively ( $\mathbf{0})$. The concentration of Au clusters in each sample is $3.06 \times 10^{-4} \%(\mathbf{O}), 1.22 \times 10^{-3} \%(\Delta), 3.67 \times 10^{-3} \%(\nabla), 6.11 \times 10^{-3} \%(\diamond), 8.56 \times 10^{-3} \%$ $(4)$.
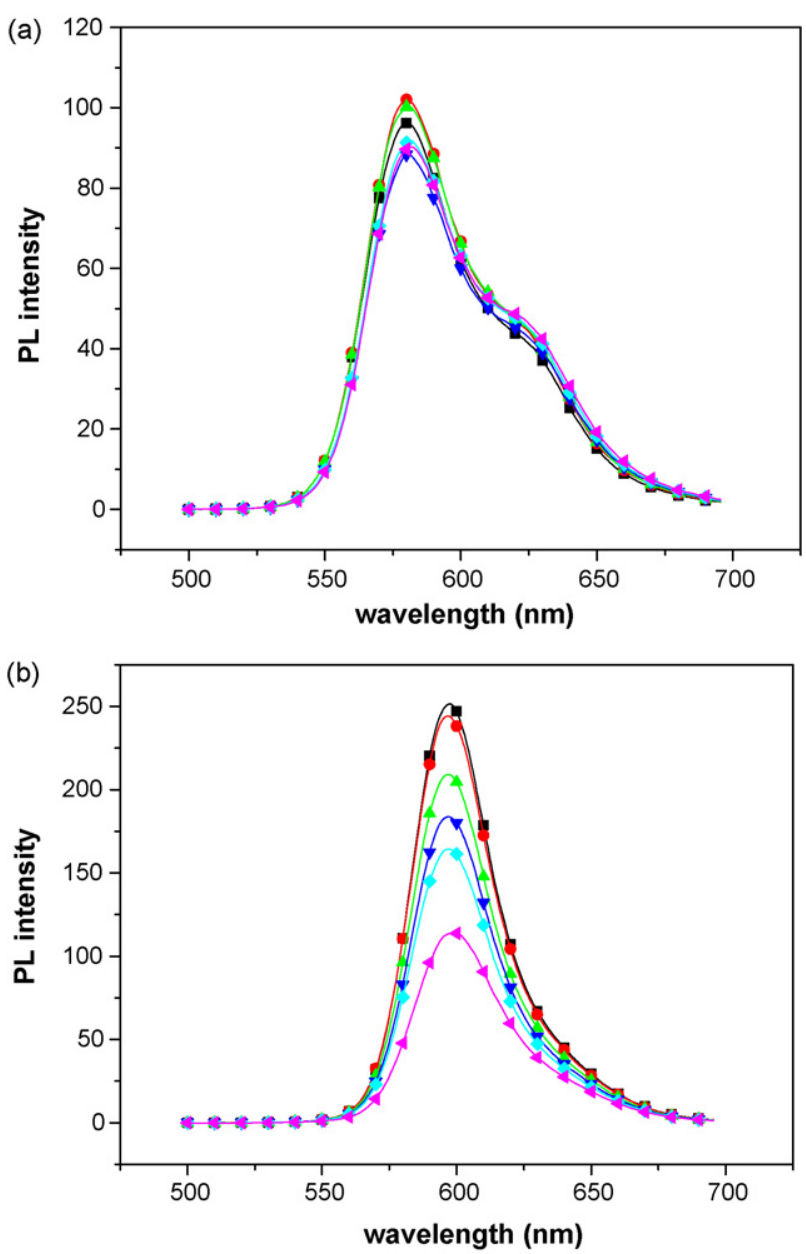

Fig. 4. PL spectra of high concentration (0.08 wt.\%) of (a) P3HT solution and (b) MEHPPV solution with or without Au nanoparticles. The black curves represent pure P3HT and MEHPPV respectively ( $\mathbf{\square})$. The concentration of Au nanoparticles in each sample is $3.06 \times 10^{-4} \%(\mathbf{O}), 1.22 \times 10^{-3} \%(\Delta), 3.67 \times 10^{-3} \%(\nabla), 6.11 \times 10^{-3} \%(\diamond)$, $8.56 \times 10^{-3} \%(4)$.

three major electronic plasmon resonance effects are expected for conducting polymer containing nanoparticles. First, the strengths of the local excitation fields are increased by the morphology and dielectric properties of Au nanoparticles through the excitation of electronic plasmon resonances. This enhancement can be explained by the metal particles ability to concentrate the intensity of local excitation. The local field induces an absorption enhancement for the polymers [11]. Second, the polymer excited state would also have an additional nonradiative decay channel when the polymer is near the nanoparticles. This damping effect arises from the out of phase dipolar component of the induced macroscopic polarization in the nanoparticle, as well as from the higher order, nonradiating induced multi-poles because the nanoparticles are in the near field of the polymer dipoles [27]. The third effect is that the nearby metal can increase the intrinsic radiative decay rate of the polymer fluorophore. The radiative yield and the lifetime of the fluorophore in the absence of a metal surface are described by $Q_{0}=\Gamma /\left(\Gamma+k_{\mathrm{nr}}\right)$ and $\left(\Gamma+k_{\mathrm{nr}}\right)^{-1}$ respectively, where $Q_{0}, \Gamma$ and $k_{\mathrm{nr}}$ are the radiative yield, radiative decay rate, and nonradiative decay rate, respectively. It follows that the radiative rate can be increased by the addition of a new rate $\Gamma_{\mathrm{m}}$ in the presence of a nearby metal surface, and the metal surface also causes the fluorophore to have another quenching effect, $k_{\mathrm{m}}$. In this case the radiative yield and lifetime of the fluorophore near the metal surface can be described 
by the following equations $[9,12,23,25]$ :

$$
Q_{\mathrm{m}}=\frac{\left(\Gamma+\Gamma_{\mathrm{m}}\right)}{\left(\Gamma+\Gamma_{\mathrm{m}}+k_{\mathrm{nr}}+k_{\mathrm{m}}\right)}
$$

$\tau_{\mathrm{m}}=\left(\Gamma+\Gamma_{\mathrm{m}}+k_{\mathrm{nr}}+k_{\mathrm{m}}\right)^{-1}$

As the value of $\Gamma_{\mathrm{m}}$ increases to exceed that of $k_{\mathrm{m}}$, the radiative yield increases while the lifetime decreases. Therefore, the emission dipole in the polymer will induce a macroscopic polarization in the nanoparticles (or clusters) which becomes large near the frequency of the electronic plasmon resonances. The in-phase dipolar component of this response increases the total emission dipole, resulting in a concomitant increase in the radiative emission rate. This electromagnetic interaction reflects the coupling of polymer dipole to the macroscopic dipole in the nanoparticle and then to the emitted radiation field.

The extinction coefficient of metallic colloids consists of two components, absorption and scattering [28]. The relative contribution from absorption and scattering depends on the type of metal, the size and the morphology of the colloids. The extent of the plasmon that can radiate into the far-field is characterized by the scattering component of the extinction $[22,26]$. Oscillating charges on the colloid surfaces induced by the incident light can radiate the energy as a far-field electromagnetic wave. Therefore, the scattering component will enhance fluorescence, while the absorption of the colloids will quench fluorescence $[22,26]$. Unlike the extinction of nanoparticles which is dominated by the absorption component, the increased extinction of aggregated colloids at long wavelengths is dominantly by the scattering component [22]. This implies that the enhanced fluorescence will occur as interacting colloids display a longer wavelength extinction. It was previously reported that aggregated colloids can display broadband and highly confined near field intensity enhancements comparable to isolated spheres $[29,30]$. They attribute an increase of emission intensity of fluorophore to an increase in the absorption efficiency caused by the field enhancements [30]. From Fig. 1, the peak of the extinction spectrum $(530 \mathrm{~nm})$ of Au nanoparticles mainly comes from the absorption component [28]. The addition of Au nanoparticles may cause the PL intensity of polymer quenching or non-enhancement. The PL intensity of P3HT remains almost the same regardless the concentration of Au nanoparticles (Fig. 4(a)). However, the PL intensity of MEHPPV is quenched by the addition of Au nanoparticles. The extent of quenching increases with the concentration of $\mathrm{Au}$ nanoparticles (Fig. 4(b)). On the other hand, the peak at $600 \mathrm{~nm}$ in Fig. 2 mostly comes from the scattering component which will enhance the PL intensity of polymer (Fig. 3). When the extinction peak of Au particles is tuned to match with the emitting range of conducting polymers, a surface plasmon resonance effect exists. The role of this resonance is to amplify the electromagnetic intensity at the location of the conducting polymers, at both the fluorescence and the incident frequencies. Because plasmon and fluorescence emitting frequency are matched, the amplified electromagnetic intensity of emitting light is much stronger than that departed from the frequency. Another contributor to this phenomenon is that matching the surface plasmon with emitting frequency induces an increase in the radiative rate of conducting polymer. Given the above reasons, amplified fluorescence intensity can be observed in the far-field.

The ratio of the fluorescence intensity observed from conducting polymers near Au colloids surfaces to that from pure conducting polymers can be described by the product of two terms as shown in below $[9,12,23-26]$ :

$Y \approx\left|L\left(\omega_{\mathrm{ex}}\right)\right|^{2} Z\left(\omega_{\mathrm{em}}\right)$ where the first term $\left|L\left(\omega_{\mathrm{ex}}\right)\right|^{2}$ describes the absorption enhancement which is proportional to the local field intensity of incident light. This term is the enhancement of local electromagnetic field intensity that approaches to the nanosized Au colloids at the excitation frequency $\omega_{\text {ex. }}$. It represents that the ability of the Au colloids concentrate the electromagnetic field when the excitation wavelength becomes resonant with the surface plasmon of the $\mathrm{Au}$ colloids. The second term $Z\left(\omega_{\mathrm{ex}}\right)$ describes the relative radiative yield of the excited conducting polymer containing Au colloids. The radiative decay rate of the coupled system consisting of Au colloids and conducting polymers is proportional to the square of the local field enhancement factor $\left|L\left(\omega_{\mathrm{em}}\right)\right|^{2}$, at the fluorescence frequency $\omega_{\mathrm{em}}$ [31]. In the coupling between the polymer dipole and the Au colloid, the Au colloid can radiate a photon before the excitation is dissipated by nonradiative pathways within the polymer. In the absence of metal colloids, the radiative yield is given as $Q_{0}=\Gamma /\left(\Gamma+k_{\mathrm{nr}}\right)$. The radiative yield is given by Eq. (2) in the presence of the Au colloids. The presence of a nearby metal surface increases the radiative rate by adding a new rate $\Gamma_{\mathrm{m}}$, but the metal surface also causes the fluorophore to have another quenching nonradiative effect, $k_{\mathrm{m}}$. The former enhances the radiative yield and the latter causes the quenching. If the polymer has a high quantum yield, the quantum yield cannot be substantially increased with the additional radiative decay rate. In this case, where energy transfers to the Au colloids, dissipation dominates, and $Z\left(\omega_{\mathrm{em}}\right)$ will be less than one. In contrast, the $Z\left(\omega_{\mathrm{em}}\right)$ will be as large as $1 / Q_{0}$ in the low quantum yield polymer [32]. This suggests that the PL intensity of polymers with low fluorescence can be increased if they are positioned near the Au colloids.

The regioregular P3HT, in which the alkyl side groups are attached to the third position of the thiophene rings in a headto-tail stereoregular order, forms thin film with nanocrystalline lamellae [33-39], resulting in relatively high hole mobilities of $0.1 \mathrm{~cm}^{2} / \mathrm{V} \mathrm{s}$ [35]. Charge carriers in the regioregular P3HT lamellae become delocalized over several polymer chains, which is consistent with the high hole mobility that has been studied by linear and nonlinear optical spectroscopies in detail [35-39]. Several groups have found evidence that photoexcitation in PPV polymers produces mainly intrachain singlet excitons [40-44]. Greenham et al. [40] found a consistent relationship between the radiative lifetime and PL quantum yield, and PL decay time for the films of several PPV derivatives, including MEHPPV, leaving no room for significant branching to an interchain species. Stagira et al. [41] reported that most of the photogenerated singlet excitons (intrachain) in the PPV film recombine to form light, and rare excitons relax to charge transfer state to transfer other chains (interchain). Rughooputh et al. [45] found that two coexisting phases are P3HT polymer in solution and in microcrystalline aggregates. Here we assume that some of P3HT polymer chains aggregate in the solution, allowing for interchain interactions. On the other hand, only intrachain interactions are present in the MEHPPV. It is possible that the excitons of pure P3HT excited by light could transfer from one chain to another chain easily and result in a lower quantum yield as compared with that of pure MEHPPV. The quantum yield of each sample is summarized in Table 1. The quantum yield of MEHPPV (0.42) is higher than that of P3HT (0.23). The results are consistent with literature data of $0.1-0.7$ [46-48]. Since the interaction of MEHPPV polymer chains is weak, the dipole in the polymer chain can recombine without much energy transferring from one chain to another. With the addition of Au nanoparticles into the MEHPPV, many dipoles in MEHPPV chains induce nanoparticles electric plasmon resonances into heat dissipation rather than re-radiative light. The value of $k_{\mathrm{m}}$ may be very large and as a result, the large value $k_{\mathrm{m}}$ in Eq. (2) reduces the quantum yield of MEHPPV. Therefore, the quantum yield of MEHPPV decreases from 0.42 to 0.31 with the addition of Au nanoparticles 
Table 1

Exciton lifetime and quantum yield of conjugated polymers with or without $\mathrm{Au}$ colloids

\begin{tabular}{lll}
\hline Sample $^{\mathrm{a}}$ & Lifetime (ns) & Quantum yield \\
\hline P3HT & 0.75 & 0.23 \\
P3HT + Au nanocluster & 0.60 & 0.57 \\
P3HT + Au nanoparticles & 0.74 & 0.22 \\
MEHPPV & 0.53 & 0.42 \\
MEHPPV + Au nanocluster & 0.45 & 0.58 \\
MEHPPV + Au nanoparticles & 0.46 & 0.31 \\
\hline
\end{tabular}

a The concentration of polymer is all kept at $0.08 \mathrm{wt} . \%$. The concentration of Au colloid is all kept at $3.67 \times 10^{-3} \mathrm{wt} . \%$.

(Table 1). On the other hand, the interaction between P3HT polymer chains is strong and the dipoles in P3HT chains are largely unaffected by the Au nanoparticles. The value of $k_{\mathrm{m}}$ coming from the adding of Au nanoparticles is unlikely to increase. The quantum yield of P3HT remains almost the same (from 0.23 to 0.22 , Table 1) after the addition of Au nanoparticles. For the Au nanoclusters added polymer systems, because the quantum yield of P3HT is small, the amount of $k_{\mathrm{nr}}$ is commeasurable to $\Gamma_{0}$. The $\Gamma_{\mathrm{m}}$ term in Eq. (2) dominates when $\Gamma_{\mathrm{m}}$ is much larger than $k_{\mathrm{m}}$ in P3HT system and the $Z\left(\omega_{\mathrm{em}}\right)$ is larger than one. From Table 1 , the quantum yield of P3HT increases from 0.23 to 0.57 with the addition of Au nanoclusters. The quantum yield of MEHPPV also increases from 0.42 to 0.58 with the addition of Au nanoclusters. The increasing of quantum yield of P3HT is much larger than that of MEHPPV. This implies that the term $Z\left(\omega_{\mathrm{em}}\right)$ for MEHPPV is smaller than that of P3HT when the Au nanoclusters are added into each polymer. Thus, the clusters can increase the PL intensity of P3HT three fold, but marginally enhance the PL intensity of MEHPPV. The exciton lifetime of P3HT and MEHPPV with either Au nanocluster or nanoparticles are measured by time resolved PL and the results are also included in Table 1 . The exciton lifetime of both polymers were decreased by adding Au nanoclusters which is directly related to an increase in $\Gamma \mathrm{m}$. However, the addition of Au nanoparticles to the polymer solution exhibited different lifetime for different polymers. The absorption effect of Au nanoparticles and the energy transfer from MEHPPV to Au nanoparticles increased the $k_{\mathrm{m}}$ and thus the lifetime of MEHPPV was decreased. In contrast, the lifetime of the P3HT system remained similar. The interaction between P3HT polymer chains is greater than that between Au nanoparticles. Thus, the energy cannot be transferred from P3HT to Au nanoparticles and has no subsequently increased $k_{\mathrm{m}}$.

Two effects of surface plasmon on fluorophore: one is the energy, transferring from the metal particles to fluorophore, which causes the PL intensity enhancement of the fluorophore; the other is the energy, transferring from the fluorophore to the metal particles, which causes the PL intensity quenching of the fluorophore. Although the scattering effect of Au nanocluster causes the increase of PL intensities of both P3HT and MEHPPV (Fig. 3), the PL intensity of polymer also is affected by its quantum yield. Because the quantum yield (Table 1 ) of MEHPPV (0.42) is higher than that of P3HT (0.23), the scattering effect of Au nanocluster on conjugated polymer which causes the PL intensity enhancement of P3HT is higher than that of MEHPPV (Fig. 3). Therefore, the energy transferring from Au nanocluster to P3HT is higher than that to MEHPPV. To prove the differences in PL phenomena between MEHPPV and $\mathrm{P} 3 \mathrm{HT}$ is due to the different quantum yield of each polymer, we tried to control its quantum yield by adjusting the polymer concentration. As the polymer concentration is diluted, its quantum yield is increased and interaction between the polymer chains decreases. The dilution also increases the free volume between polymer chains and the transfer of excited electrons between the polymer chains is diminished. Thus, the dipole will be confined in one polymer chain without any transformation. This allows us to increase the quantum yield by diluting the polymer concentrations. The concentrations of MEHPPV were diluted and kept at $1.48 \times 10^{-5} \mathrm{wt}$.\% in toluene. The quantum yield is calculated according to Eq. (1) by using DCM dye as a standard and it is increased to 0.84 . The quantum efficiency of many organic molecules can be close to $100 \%$ in dilute solutions. There have been reported on laser emission from MEHPPV diluted solution (the quantum yield is about 1 ) and from the diluted blend film of MEHPPV and polystyrene, where the polymer chains are isolated $[49,50]$. The higher efficiency of the radiative recombination will be when the polymer molecules are more separated spatially in the more dilute solution [49]. The concentrations of $\mathrm{Au}$ nanoparticles clusters in the polymer solution were $3.06 \times 10^{-4}$, $1.22 \times 10^{-3}, 3.67 \times 10^{-3}, 6.11 \times 10^{-3}$, and $8.56 \times 10^{-3}$ wt.\% respectively as before. The PL results are shown in Fig. 5(a). We observed the PL quenching phenomena as we using high quantum yield of dilute MEHPPV solution. The quantum yield of MEHPPV with the addition of Au nanoclusters $\left(3.67 \times 10^{-3} \%\right)$ is 0.57 . Most MEHPPV chain is surrounded by solvent molecules (toluene) before the adding of Au colloids. However, after the adding of the Au nanoclusters, the environment of each MEHPPV chain will be surrounded by many Au colloids. Although the scattering effect of Au nanoclusters cause the PL intensity enhancement, each polymer chain is surrounded by Au colloids, most of dipoles in the MEHPPV chains
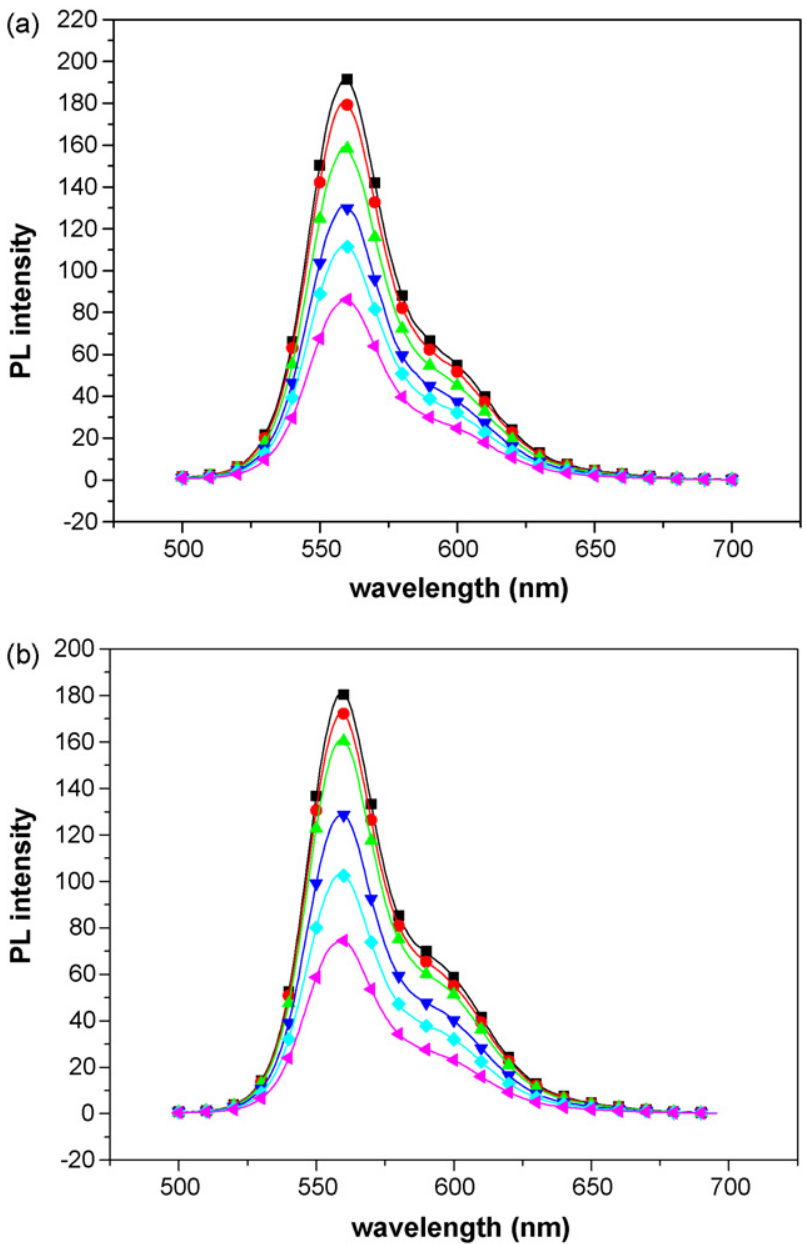

Fig. 5. PL spectra of low concentration $\left(1.48 \times 10^{-5}\right.$ wt.\%) of (a) MEHPPV solution and (b) P3HT solution with or without Au clusters. The black curves represent pure MEHPPV and P3HT respectively ( $\mathbf{\square})$. The concentration of Au clusters in each sample is $3.06 \times 10^{-4} \%(\mathbf{O}), 1.22 \times 10^{-3} \%(\Delta), 3.67 \times 10^{-3} \%(\nabla), 6.11 \times 10^{-3} \%(\diamond), 8.56 \times 10^{-3} \%$ $(<)$. 
can induce the nanoparticles to form plasmon resonances, and then further dissipate into heat. Energy transfer from polymer to Au nanocluster is higher than that from Au nanocluster to polymer, thus the PL intensity is quenched. Therefore, we expect the $k_{\mathrm{m}}$ in Eq. (2) of dilute MEHPPV is larger than that of high concentration MEHPPV (0.08 wt.\%), thus $k_{\mathrm{m}}$ is larger than $\Gamma_{\mathrm{m}}$. From Eq. (2), the ratio, $\mathrm{Qm}_{\mathrm{m}}$, decreased as we diluted the MEHPPV concentrations and accordingly, we found the PL quenching phenomena (Fig. 5(a)). The result is agreed with literature. If the fluorophore has a high quantum yield, the additional radiative decay rate $\left(\Gamma_{\mathrm{m}}\right)$ cannot substantially increase the quantum yield. Thus, the energy transfer quenching to the metal will dominate [9]. The same experiment was done for the P3HT system and the quenching effect was also observed (Fig. 5(b)). The quantum yield of dilute P3HT $\left(1.48 \times 10^{-5} \%\right)$ is 0.82 that is decreased to 0.58 after the addition of Au nanoclusters $\left(3.67 \times 10^{-3} \%\right)$. As described above, the PL quenching phenomena could be seen both from the increasing of quantum yield of P3HT and mismatching the emitting range with the plasmon resonances.

\section{Conclusion}

We have studied the optical relationship between conducting polymers and Au colloids. Two mechanisms, surface plasmons resonance and energy transfer from conducting polymer to Au colloids, control the PL property of polymer. The addition of Au nanoparticle colloids into the polymer solution increases the absorption rate and the emitting rate of conducting polymer. When the emitting frequency of the conducting polymer matches the electric plasmons scattering frequency of Au nanocluster colloids, the emitting intensity of the conducting polymer is enhanced. In contrast, quenching of the conducting polymer emission is observed when the energy from the conducting polymer is absorbed by Au nanoparticle colloids, without radiating into far-field. Therefore, we can modulate the PL intensity of conducting polymer by adjusting the polymer concentration, adjusting the morphology of the colloids or by using the combination of both factors.

\section{Acknowledgments}

We acknowledge Ming-Chung Wu and Chih-Min Chuang for taking the TEM images. This work was supported by the National Science of Council of Republic of China (NSC96-2628-E-002-017MY3) and US Air Force (AOARD 07-4014). Mr. An-Jey Su of University of Pittsburgh helped to edit this manuscript.

\section{References}

[1] K. Kneipp, H. Kneipp, I. Itzkan, R.R. Dasari, M.S. Feld, Chem. Rev. 99 (1999) 2957-2975.

[2] Y. Cao, R. Jin, C. Mirkin, Science 297 (2002) 1536-1540.

[3] K. Kneipp, Y. Wang, H. Kneipp, L. Perelman, I. Itzkan, R. Dasari, M. Feld, Phys. Rev. Lett. 78 (1997) 1667-1670.

[4] S. Nie, S. Emery, Science 275 (1997) 1102-1106.
[5] C.E. Talley, J.B. Jackson, C. Oubre, N.K. Grady, C.W. Hollars, S.M. Lane, T.R. Huser, P. Nordlander, N.J. Halas, Nano Lett. 5 (2005) 1569-1574.

[6] S. Lefrant, I. Baltog, M.L. de la Chapelle, M. Baibarac, G. Louarn, C. Journet, P. Bernier, Synth. Met. 100 (1999) 13-27.

[7] E. Dulkeith, M. Ringler, T.A. Klar, J. Feldmann, A.M. Javier, W.J. Parak, Nano Lett. 5 (2005) 585-589.

[8] Y. Chen, K. Munechika, D.S. Ginger, Nano Lett. 7 (2007) 690-696.

[9] J.R. Lakowicz, Anal. Biochem. 298 (2001) 1-24.

[10] T. Nakamura, S. Hayashi, Jpn. J. Appl. Phys. 44 (2005) 6833-6837.

[11] D.A. Weitz, S. Garoff, J.I. Gersten, A. Nitzan, J. Chem. Phys. 78 (1983) 5324-5338.

[12] J.R. Lakowicz, Y. Shen, S. D'Auria, J. Malicka, J. Fang, Z. Gryczynski, I. Gryczynski, Anal. Biochem. 301 (2002) 261-277.

[13] T. Hayakawa, S.T. Selvan, M. Nogami, Appl. Phys. Lett. 74 (1999) 1513-1515.

[14] J.H. Song, T. Atay, S. Shi, H. Urabe, A.V. Kurmikko, Nano Lett. 5 (2005) 1557-1561.

[15] J. Lee, A.O. Govorov, J. Dulka, N.A. Kotov, Nano Lett. 4 (2004) 2323-2330.

[16] H.J. Park, D. Vak, Y.Y. Noh, B. Lim, D.Y. Kim, Appl. Phys. Lett. 90 (2007) 1611071-1611073.

[17] M. Green, P. O’Brien, Chem. Commun. (2000) 183-184.

[18] J.V. Morris, M.A. Mahaney, J.R. Huber, J. Phys. Chem. 80 (1976) 969-974.

[19] D.C. Easter, A.P. Baronavski, Chem. Phys. Lett. 201 (1993) 153-158.

[20] R.L. Chern, X.X. Liu, C.C. Chang, Phys. Rev. E 76 (2007) 0166091-0166099.

[21] C.L. Haynes, A.D. McFarland, L.L. Zhao, R.P. Van Duyne, G.C. Schatz, J. Phys. Chem. B 107 (2003) 7337-7342.

[22] C.G. Blatchford, J.R. Campbell, J.A. Creighton, Surf. Sci. 120 (1982) 435-455.

[23] E.M. Goldys, A. Barnett, F. Xie, K. Drozdowicz-Tomsia, I. Gryczynski, E.G. Matveeva, Z. Gryczynski, T. Shtoyko, Appl. Phys. A 89 (2007) 265-271.

[24] K. Sokolov, G. Chumanov, T.M. Cotton, Anal. Chem. 70 (1998) 3898-3905.

[25] J. Zhang, E. Matveeva, I. Gryczynski, Z. Leonenko, J.R. Lakowicz, J. Phys. Chem. B 109 (2005) 7969-7975.

[26] J.R. Lakowicz, Anal. Biochem. 337 (2005) 171-194.

[27] M. Martin, Rev. Mod. Phys. 57 (1985) 783-826.

[28] J. Yguerabide, E.E. Yguerabide, Anal. Biochem. 262 (1998) 137-156.

[29] S. Foteinopoulou, J.P. Vigneron, C. Vandenbem, Opt. Express 15 (2007) 4253-4267.

[30] M.A. Noginov, G. Zhu, M. Bahoura, C.E. Small, C. Davison, J. Adegoke, V.P. Drachev, P. Nyga, V.M. Shalaev, Phys. Rev. B 74 (2006) 1842031-1842038.

[31] A. Wokaun, Solid State Phys. 38 (1984) 223-294.

[32] J. Kummerlen, A. Leitner, H. Brunner, F.R. Aussenegg, A. Wokaun, Mol. Phys. 80 (1993) 1031-1039.

[33] S. Hugger, R. Thomann, T. Heinzel, T.T. Albrecht, Colloid Polym. Sci. 289 (2004) 932-938.

[34] T.Q. Nguyen, V. Doan, B.J. Schwatz, J. Chem. Phys. 110 (1999) 4068-4078.

[35] H. Siminghaus, P.J. Brown, R.H. Friend, M.M. Nielsen, K. Bechgaard, B.M.W. Langeveld-Voss, A.J.H. Spiering, R.A.J. Janssen, E.W. Meijer, P. Herwig, D.M. de Laeeuw, Nature 401 (1999) 685-688.

[36] B. Xu, S. Holdcroft, Macromolecules 26 (1993) 4457-4460.

[37] R. Osterbacka, C.P. An, X.M. Jiang, Z.V. Vardeny, Science 287 (2000) 839-842.

[38] X.M. Jiang, C.P. An, R. Osterbacka, Z.V. Vardeny, Synth. Met. 116 (2001) 203-206

[39] R. Osterbacka, C.P. An, X.M. Jiang, Z.V. Vardeny, Synth. Met. 116 (2001) 317-320.

[40] N.C. Greenham, I.D.W. Samuel, G.R. Hayes, R.T. Phillips, Y.A.R.R. Kessener, S.C. Moratti, A.B. Holmes, R.H. Friend, Chem. Phys. Lett. 241 (1995) 89-96.

[41] S. Stagira, M. Nisoki, G. Lanzani, S.D. Silvestri, T. Cassano, R. Tommasi, F. Babudri, G.M. Farinola, F. Naso, Phys. Rev. B 64 (2001) 2052051-2052058.

[42] S.V. Frolov, M. Liess, P.A. Lane, W. Gellermann, Z.V. Vardeny, M. Ozaki, K. Yoshino, Phys. Rev. Lett. 78 (1997) 4285-4288.

[43] N.T. Harrison, G.R. Hayes, R.T. Phillips, R.H. Friend, Phys. Rev. Lett. 77 (1996) $1881-1884$.

[44] G.R. Hayes, I.D.W. Samuel, R.T. Phillips, Synth. Met. 84 (1997) 889-890.

[45] S.D.D.V. Rughooputh, S. Hotta, A.J. Heeger, F. Wudl, J. Polym. Sci. B: Polym. Phys 25 (1987) 1071-1078.

[46] I.D.W. Samuel, B. Crystall, G. Rumbles, P.L. Burn, A.B. Holmes, R.H. Friend, Chem Phys. Lett. 213 (1993) 472-478.

[47] Y.F. Huang, H.L. Chen, J.W. Ting, C.S. Liao, R.W. Larsen, W.S. Fann, J. Phys. Chem. B 108 (2004) 9619-9622.

[48] B. Pal, W.C. Yen, J.S. Yang, W.F. Su, Macromolecules 40 (2007) 8189-8194.

[49] D. Moses, Appl. Phys. Lett. 60 (1992) 3215-3216.

[50] F. Hide, B.J. Schwartz, M.A. Diaz-Garcia, A.J. Heeger, Chem. Phys. Lett. 256 (1996) 424-430. 